\title{
A Solution for the Generalized Synchronization of a Class of Chaotic Systems Based on Output Feedback
}

\author{
Carlos Aguilar-Ibanez, ${ }^{1}$ R. Martínez-Guerra, ${ }^{2}$ C. Pérez-Pinacho, ${ }^{2}$ \\ E. García-Canseco, ${ }^{3}$ and Miguel S. Suarez-Castanon ${ }^{4}$ \\ ${ }^{1}$ Instituto Politécnio Nacional-CIC, Avenida Juan de Dios Bátiz, s/n, 07738 México, DF, Mexico \\ ${ }^{2}$ Department of Automatic Control, CINVESTAV-IPN, Avenida Instituto Politécnico Nacional 2508, 07360 México, DF, Mexico \\ ${ }^{3}$ Faculty of Sciences, Autonomous University of Baja California, Km. 103 Carretera Tijuana-Ensenada, 22860 Ensenada, BCN, Mexico \\ ${ }^{4}$ Instituto Politécnio Nacional-ESCOM, Avenida Juan de Dios Bátiz Esquina Miguel Othón de Mendizábal, 07738 México, DF, Mexico
}

Correspondence should be addressed to Carlos Aguilar-Ibanez; carlosaguilari@cic.ipn.mx

Received 9 June 2015; Revised 4 September 2015; Accepted 6 September 2015

Academic Editor: Rafael Morales

Copyright (C) 2015 Carlos Aguilar-Ibanez et al. This is an open access article distributed under the Creative Commons Attribution License, which permits unrestricted use, distribution, and reproduction in any medium, provided the original work is properly cited.

A solution to the output-feedback generalized synchronization problem for two chaotic systems, namely, the master and the slave, is presented. The solution assumes that the slave is controlled by a single input, and the states of each system are partially known. To this end, both systems are expressed in their corresponding observable generalized canonical form, through their differential primitive element. The nonavailable state variables of both systems are recovered using a suitable Luenberger observer. The convergence analysis was carried out using the linear control approach in conjunction with the Lyapunov method. Convincing numerical simulations are presented to assess the effectiveness of the obtained solution.

\section{Introduction}

The synchronization of strictly different chaotic systems is a current challenge in Control Theory. In general, accomplishing and understanding this kind of synchronization, referred to as generalized synchronization (GS), are a difficult problem and are very important because its solution can be useful to actual applications. For instance, chaotic signals have been used in secure information transmission. In this regard, interesting secure communication methods based on GS can be found in the literature. In [1], a channelindependent chaotic secure communication scheme based on GS is proposed. A similar scheme, also based on GS, with a remarkable stability to noise and numerically validated using the Rössler system is developed in [2]. Two novel generalized chaos synchronization based secure communication schemes are introduced in $[3,4]$. The first scheme is complemented with a transposition function and is tested using the Chen chaotic circuit; the second scheme is oriented to encrypt images and uses a theorem introduced by the authors, which is a generalization of GS to an array of differential equations. From a theoretical point of view, synchronization of strictly different chaotic systems helps to explain how some oscillatory systems, like the intrinsic neuron model, act cooperatively to develop tasks or solve problems [5]. This behavior also occurs in nature and can be responsible for the transition to low-dimensional behavior in systems with many degrees of freedom. A full review of GS is beyond the scope of this study; however, we mention two seminal works. In [6] the authors developed a method for decomposing chaotic systems such that linear GS can be achieved. Finally, we mention work [5], where the authors proposed a method used to detect and study generalized synchronization in driveresponse systems. The method uses an identical response system to monitor the synchronized motions. We suggest to the interested reader the following list of remarkable works [7-16].

Loosely speaking the GS phenomenon occurs when the trajectories of one system, through a functional mapping, are equal to trajectories of another. In other words, let us suppose 
that we have two systems, $\Sigma_{m}$ and $\Sigma_{s, u}$, named, respectively, master and slave, with their corresponding trajectories, $\mathbf{x}_{m}(t)$ and $\mathbf{x}_{s}(t)$ [17]. That is, GS is accomplished [18] if there exists a differential primitive element that generates a mapping $H_{m s}$ from the trajectories $\mathbf{x}_{m}(t)$ of $\Sigma_{m}$ in the algebraic manifold $M$ to the trajectories $x_{s}(t)$ of $\Sigma_{s, u}$ in its space $S$, in which case it holds that $H_{m s}\left(\mathbf{x}_{s}(t)\right)=\mathbf{x}_{m}(t)$. It is noteworthy that for identical systems the functional mapping $H_{m s}$ corresponds to the identity [19]. In our case, we assume that the behavior of the slave system $\Sigma_{s, u}$ can be either chaotic or bounded and oscillatory. Evidently, to accomplish GS there must be a control action acting over the slave $\Sigma_{s, u}$ (for simplicity, symbols $\Sigma_{m}$ and $\Sigma_{s, u}$ refer to the uncontrolled master system and the controlled slave system, resp. In the same way, $\mathbf{x}_{m}$ and $\mathbf{x}_{s}$, are, resp., the states of systems $\Sigma_{m}$ and $\Sigma_{s, u}$.)

In this work, we solve the output-feedback GS problem for systems $\Sigma_{m}$ and $\Sigma_{s, u}$, when only a single output of each system is available or measurable, and only a single controller is used in the slave system. This restriction makes the GS problem more challenging and interesting than the works mentioned above. To solve it, the nonavailable state variables of systems $\Sigma_{m}$ and $\Sigma_{s, u}$ must be reconstructed from the measurable outputs. To overcome this issue, we exploit some ad hoc differential algebraic properties found in some chaotic systems, by choosing the differentiable primitive element [20]. This primitive element allows us to express both systems in a generalized canonical form. Thence, we solve the GS problem as if it were an instance of the trajectorytracking problem. To this end, it is necessary to split the slave controller into two parts: one devoted to feedback the output tracking error between the master and the slave systems and the other compensating for the underlying and nonavailable state variables of each system. We underscore that our control design basically consists of finding a suitable differentiable primitive element to express the master and slave systems in their corresponding canonical form, in conjunction with a kind of Luenberger observer to reconstruct the nonavailable variable states. Fortunately, there are in nature several chaotic systems that can be expressed in this form.

The remainder of this paper is organized as follows. In Section 2, we introduce some concepts necessary to establish the problem statement properly. In Section 3, we solve the output-feedback GS control problem. Numerical experiments to assess the effectiveness of our solution are presented in Section 4. The final remarks are given in Section 5.

Notation. To simplify the development of this work, we adopt the following notation:

$$
A_{i}=\left[\begin{array}{cccccc}
0 & 1 & 0 & 0 & \cdots & 0 \\
0 & 0 & 1 & 0 & \cdots & 0 \\
\vdots & \vdots & \vdots & \vdots & \vdots & \vdots \\
0 & 0 & \cdots & \cdots & 0 & 1 \\
0 & 0 & 0 & 0 & \cdots & 0
\end{array}\right] \in \mathbb{R}^{i \times i}
$$

$$
\begin{gathered}
B_{i}=\left[\begin{array}{c}
0 \\
\vdots \\
0 \\
1
\end{array}\right] \in \mathbb{R}^{i} ; \\
C_{i}=\left[\begin{array}{c}
1 \\
\vdots \\
0 \\
0
\end{array}\right] \in \mathbb{R}^{i} .
\end{gathered}
$$

\section{Preliminaries}

Before proceeding, we introduce some needful concepts [2023]. From the theorem of differential primitive element [20] we posit a single element $\delta \in L$, which is a differential primitive element, such that $L=K(\delta)$; that is, $L$ is differentially generated by $K$ and $\delta$ ( $K$ and $L$ are differential fields).

Consider the following nonlinear system:

$$
\dot{\mathbf{x}}=F(\mathbf{x}, u),
$$

where $\mathbf{x} \in \mathbb{R}^{n}$ is the system state and $u$ is the system input. Also, suppose that there exists an auxiliary variable $\bar{y}$ (differential primitive element), defined as

$$
\bar{y}=\sum_{i} \alpha_{i} x_{i}, \quad \alpha_{i} \in \mathbb{R}
$$

which allows us to write system (2) in the following form:

$$
\dot{\xi}=A_{n} \xi+B_{n}(-\mathfrak{E}(\xi)+p u)
$$

where $\xi=\left[\xi_{1}, \xi_{2}, \ldots, \xi_{n}\right]^{T}$ with $\xi_{i}=\bar{y}^{(i-1)}$, for $i=\{1, \ldots, n\}$, $\mathfrak{E}(\cdot)$ is a scalar nonlinear function of their arguments and may not be defined everywhere, and $p$ is the gain constant of the single input of $u$. The above system representation is well known as the generalized controller canonical form, and variable $\bar{y}$ is the measurable output of the system $[21,24]$.

2.1. Problem Statement. In this section we introduce formally the main problem of this work, which consists of solving the output-feedback GS problem for two chaotic systems, in a master-slave configuration, where the states of both systems are partially known. To this end, we propose a suitable definition for the GS problem, based on previous works [21]. It should be noted that our definition uses the notion of differential primitive element, already mentioned.

Let us consider the following two nonlinear systems in a master-slave configuration, where the master system, $\Sigma_{m}$, is given by

$$
\begin{aligned}
& \dot{\mathbf{x}}_{m}=F_{m}\left(\mathbf{x}_{m}\right) ; \\
& y_{m}=h_{m}\left(\mathbf{x}_{m}\right),
\end{aligned}
$$


and the controlled slave system, $\Sigma_{s, u}$, is defined by

$$
\begin{aligned}
& \dot{\mathbf{x}}_{s}=F_{s}\left(\mathbf{x}_{s}, u\left(\mathbf{x}_{s}, \mathbf{x}_{m}\right)\right) ; \\
& y_{s}=h_{s}\left(\mathbf{x}_{s}\right),
\end{aligned}
$$

where $\mathbf{x}_{s}=\left(x_{1_{s}}, \ldots, x_{n_{s}}\right) \in \mathbb{R}^{n}, \mathbf{x}_{m}=\left(x_{1_{m}}, \ldots, x_{n_{m}}\right) \in \mathbb{R}^{n}$, $h_{s}: \mathbb{R}^{n} \rightarrow \mathbb{R}, h_{m}: \mathbb{R}^{n} \rightarrow \mathbb{R}, u: \mathbb{R}^{n} \times \mathbb{R}^{n} \rightarrow \mathbb{R}, y_{m}$, and $y_{s} \in \mathbb{R} . F_{s}, F_{m}, h_{s}$, and $h_{m}$ are assumed to be polynomial in their arguments. Before introducing the GS definition, we need to introduce the following two important assumptions, related to some structural properties of $\Sigma_{m}$ and $\Sigma_{s, u}$ and based on the result found in [21].

Assumption A1. Suppose that the master system, $\Sigma_{m}$, given in (5), can be written as

$$
\dot{\mathbf{z}}_{m}=A_{n} \mathbf{z}_{m}+B_{n}\left(-\mathfrak{E}_{m}\left(\mathbf{z}_{m}\right)\right)
$$

where $\mathbf{z}_{m}=\left[z_{m_{1}}, \ldots, z_{m_{n}}\right]^{T}=\Phi_{m}\left(\mathbf{x}_{m}\right)$ is the vector of function, with $z_{m_{k}}=y_{m}^{(k-1)}$ for $k=\{1, \ldots, n\}, y_{m}$ is a differential primitive element for the master system, and $£_{m}(\cdot)$ is a scalar nonlinear function. Additionally, the trajectories of free system (7) must be forward complete in some compact set in $\mathbb{R}^{n}$.

Assumption A2. Suppose that the slave system $\Sigma_{s, u}$, given in (6), can be written as

$$
\dot{\mathbf{z}}_{s}=A_{n} \mathbf{z}_{s}+B_{n}\left(-\mathfrak{E}_{s}\left(\mathbf{z}_{s}\right)+p u\right),
$$

where $\mathbf{z}_{s}=\left[z_{s_{1}}, \ldots, z_{s_{n}}\right]^{T}=\Phi_{s}\left(\mathbf{x}_{s}\right)$ with $z_{s_{k}}=y_{s}^{(k-1)}$, for $k=$ $\{1, \ldots, n\}, y_{s}$ is a differential primitive element for the slave system, $p$ is a gain constant of the single input $u$, and $\mathfrak{E}_{s}(\cdot)$ is a scalar nonlinear function.

Notice that the functions vectors, $\Phi_{m}, \Phi_{s}: \mathbb{R}^{n} \rightarrow$ $\mathbb{R}^{n}$, are generated through the corresponding differentiable primitive element. This construction allows us to solve straightforwardly the GS problem. Finally, we mention that constructions (7) and (8) are in agreement with the canonical form previously introduced in $[23,25]$. Now we can introduce the main definition of this study, as follows.

Definition 1 (generalized synchronization). Let us consider systems, $\Sigma_{m}$ and $\Sigma_{s, u}$, under Assumptions A1 and A2, with $\Phi_{s}$ being invertible. The slave and master systems are said to be in a state of GS if there exists a differential primitive element that generates a transformation $H_{m s}: \mathbb{R}^{n} \rightarrow \mathbb{R}^{n}$ with $H_{m s}=\Phi_{s}^{-1} \circ \Phi_{m}$ and there exist an algebraic manifold $M=\left\{\left(x_{s}, x_{m}\right) \mid x_{m}=H_{m s}\left(x_{s}\right)\right\}$ and a compact set $B \subset$ $\mathbb{R}^{n} \times \mathbb{R}^{n}$ with $M \subset B$ such that their trajectories with initial conditions in $B$ approach $M$ as $t \rightarrow \infty$. This definition leads to the following criterion:

$$
\lim _{t \rightarrow \infty}\left\|H_{m s}\left(x_{s}\right)-x_{m}\right\|=0 .
$$

Remark 2. As far as we know, the GS problem has been solved assuming that states $\left(x_{s}, x_{m}\right)$ are available for measurement. In this work, we moved a step forward, by considering the case when the states of both systems are partially known. To this end, the proposed controller is based on outputfeedback stabilization, instead of using full-state control. It is important, because in several actual applications the whole state is not available or is unpractical or impossible to measure. For instance, in the classical Duffing system only the position is available. On the other hand, finding a general solution of this problem is rather difficult.

As it can be seen original systems (5) and (6) are expressed in their corresponding observable generalized canonical form, through their corresponding differential primitive element. It is worthy to mention that, fortunately, several chaotic systems can be expressed in such observable generalized canonical form. Now, if Assumptions A1 and A2 hold, there exists a full-state feedback controller, $u\left(\mathbf{z}_{m}, \mathbf{z}_{s}\right)$, such that $\lim _{t \rightarrow \infty}\left\|\mathbf{z}_{m}-\mathbf{z}_{s}\right\|=0$ [21]. We underscore that this result assumes that the whole state of both systems, the master and the slave, is available for measurements.

Now, we can introduce the main problem of this work.

Problem Statement. Consider the uncontrolled master chaotic system (7) and the controlled slave chaotic system (8), satisfying, respectively, Assumptions $\mathrm{A} 1$ and $\mathrm{A} 2$, and the vector function $\Phi_{s}^{-1}$ existing at least locally. The main goal consists of proposing the slave system controller:

$$
u\left(y_{s}, \widehat{y}_{s}^{(1)}, \ldots, \widehat{y}_{s}^{(n)}, y_{m}, \ldots, \widehat{y}_{m}^{(n)}\right),
$$

which solves the GS problem, where $\widehat{y}^{(i)}$ are estimations of the unknown $y^{(i)}$.

As this shows, we are dealing with a more difficult problem configuration. Contrary to several previous works $[5,6,26]$, we do not use any control action over the master chaotic system, while the slave controller only uses one measurable output from the slave and one from the master. This problem instance can be considered more appealing, because it resembles several actual implementations. On the other hand, it is less expensive to control a system using state estimations than using actual measurements.

To solve this problem, it is necessary to reconstruct the nonavailable dynamics of both systems, the master and the slave. Therefore, we introduce a suitable version of the assumption introduced in [21].

Assumption P1. Suppose that there exists a primitive element, $y_{m}$, of the master system " $\Sigma_{m}$ " (5) that transforms it into the new form " $\Sigma_{m_{T}}$ ", given by

$$
\dot{\mathbf{z}}_{m}=A_{n} \mathbf{z}_{m}+B_{n}\left(\phi_{m}\left(y_{m}\right)+K_{m}^{T} \mathbf{z}_{m}+f_{m}\left(\mathbf{z}_{m}\right)\right)
$$

where $\mathbf{z}_{m}=\Phi_{s}\left(\mathbf{x}_{m}\right), \phi_{m}$ is a scalar nonlinear function that depends on the output $y_{m}, K_{m}$ is a vector of constants, and $f_{m}(\mathbf{z})$ is a scalar Lipschitz function in $\mathbf{z}$ on the open set $D \subset$ $\mathbb{R}^{n}$. That is,

$$
\left\|f_{m}(\mathbf{x})-f_{m}(\mathbf{y})\right\| \leq \gamma_{m}\|\mathbf{x}-\mathbf{y}\| ; \quad \forall \mathbf{x}, \mathbf{y} \in D
$$


Assumption P2. Suppose that there exists a primitive element, $y_{s}$, of the slave system " $\Sigma_{s, u}$ " (6) that transforms it into the new form " $\Sigma_{s_{T}, u}$ ", given by

$$
\dot{\mathbf{z}}_{s}=A_{n} \mathbf{z}_{s}+B_{n}\left(\phi_{s}\left(y_{s}\right)+K_{s}^{T} \mathbf{z}_{s}+f_{s}\left(\mathbf{z}_{s}\right)+p u\right),
$$

where $\mathbf{z}_{s}=\Phi_{s}\left(\mathbf{x}_{s}\right), \phi_{s}$ is a scalar nonlinear function that depends on the output $y_{s}, K_{s}$ is a constant vector, and $f_{s}\left(\mathbf{z}_{s}\right)$ is a scalar Lipschitz function in $\mathbf{z}$, with its corresponding constant $\gamma_{s}$. Finally, $p \neq 0$ is a fixed constant and $u$ is the single input of the system.

Comment 1. Assumptions A1 and A2 are needed to solve the GS problem, when the states of both systems, the master and the slave, are known. Assumptions P1 and P2 are needed to solve the GS problem when only a single output of each system can be measurable. As we can see, our goal is to move a step forward with respect to the previous works. On the other hand, there are many chaotic systems that admit the representation, given by (11) and (13).

\section{Solving the GS Control Problem for a Class of Chaotic Nonlinear Systems}

In this section we present a solution to the main problem of this study. To this end, we design a Lyapunov-based observer to recover the underlying dynamics of the master and slave systems, $\Sigma_{m}$ and $\Sigma_{s, u}$. Afterwards, using the recovered dynamics by a static feedback $u$, we solve the GS problem.

Reconstructing the Nonavailable Dynamic of $\Sigma_{m}$ or $\Sigma_{s, u}$. Suppose that we have a nonlinear system, defined by

$$
\begin{aligned}
& \dot{\mathbf{z}}=A_{n} \mathbf{z}+B_{n}\left(\phi(y)+f(\mathbf{z})+K^{T} \mathbf{z}+p u\right), \\
& y=C_{n}^{T} \mathbf{z},
\end{aligned}
$$

where $y$ is the single measurable output, $\mathbf{z} \in \mathbb{R}^{n}$ is the partially known state, $\phi(y)$ is any scalar function, and $f(\mathbf{z})$ is a scalar Lipschitz function in $\mathbf{z} \in \mathbb{R}^{n}$, with $\gamma$ as the Lipschitz constant, and $K \in \mathbb{R}^{n}$ constant. Now, consider the observer of this nonlinear system, defined as

$$
\begin{aligned}
\dot{\mathbf{z}}= & A_{n} \widehat{\mathbf{z}}+B_{n}\left(\phi\left(y_{m}\right)+f(\widehat{\mathbf{z}})+K^{T} \widehat{\mathbf{z}}+p u\right) \\
& -L_{n_{\kappa}}\left(C_{n}^{T} \widehat{\mathbf{z}}-y\right)
\end{aligned}
$$

where $\widehat{\mathbf{z}}$ is an estimation of $\mathbf{z}, L_{n_{\kappa}}=\left[\kappa_{1}, \kappa_{2}, \ldots, \kappa_{n}\right]^{T} \in \mathbb{R}^{n}$ is a vector of constants, and $u$ is the system input. Notice that system (14) has a similar structure to that of the systems defined in Assumptions P1 and P2. The following proposition provides sufficient conditions to ensure that the estimation error $\widetilde{\mathbf{z}}=\mathbf{z}-\widehat{\mathbf{z}}$ converges to zero, as long as $t \rightarrow \infty$.

Proposition 3. Consider systems (14) and (15). If the vector of constants, $L_{n_{\kappa}}$, is selected, according to

$$
P_{n_{\kappa}} M_{n_{\kappa}}+M_{n_{\kappa}}^{T} P_{n_{\kappa}}=-I_{n}
$$

where $M_{n_{\kappa}}=\left(A_{n}+B_{n} K^{T}-L_{n_{\kappa}} C_{n}^{T}\right)$ and $P_{n_{\kappa}}>0$ satisfying

$$
2 \gamma\left\|\bar{r}_{n}\right\|<1
$$

where $\bar{r}_{n}=P_{n_{\kappa}} B_{n}\left(\bar{r}_{n}\right.$ is in fact the last row of matrix $\left.P_{n_{\kappa}}\right)$, then, the error $\widetilde{\mathbf{z}}=\mathbf{z}-\widehat{\mathbf{z}}$ converges asymptotically and exponentially to zero.

Proof. From (11) and (15), it is easy to show that the dynamic of the error $\widetilde{\mathbf{z}}$ is given by

$$
\dot{\tilde{\mathbf{z}}}=\left(A_{n}+B_{n} K^{T}-L_{n_{\kappa}} C_{n}^{T}\right) \widetilde{\mathbf{z}}+B_{n}(f(\mathbf{z})-f(\widehat{\mathbf{z}})) .
$$

In order to analyze the convergence of the state $\widetilde{\mathbf{z}}$, we propose the Lyapunov function $V(\widetilde{\mathbf{z}})=\widetilde{\mathbf{z}}^{T} P_{n_{\kappa}} \widetilde{\mathbf{z}}$, where $P_{n_{\kappa}}=P_{n_{\kappa}}^{T}>0$, whose time derivative, along of (18), is given by

$$
\dot{V}(\widetilde{\mathbf{z}})=-\|\widetilde{\mathbf{z}}\|^{2}+2 \widetilde{\mathbf{z}} P_{n_{\kappa}} B_{n}(f(\mathbf{z})-f(\widehat{\mathbf{z}})) .
$$

Now, it is quite easy to see that the following inequality holds:

$$
\left|\widetilde{\mathbf{z}} P_{n_{\boldsymbol{\kappa}}} B_{n}(f(\mathbf{z})-f(\widehat{\mathbf{z}}))\right| \leq \gamma\left\|P_{n_{\kappa}} B_{n}\right\|\|\widetilde{\mathbf{z}}\|^{2} .
$$

Therefore, from (19) and (20), we have that $\dot{V}$ can be upper bounded, as

$$
\dot{V}(\widetilde{\mathbf{z}}) \leq-\|\widetilde{\mathbf{z}}\|^{2}\left(1-2 \gamma\left\|\bar{r}_{n}\right\|\right) .
$$

Evidently, $\dot{V}<0$, for all $\widetilde{\mathbf{z}} \neq 0$, in the case that inequality (17) is fulfilled.

The following remark allows us to propose the needed observers for $\Sigma_{m_{T}}$ and $\Sigma_{s_{T}, u}$.

Remark 4. Assumptions $\mathrm{P} 1$ and $\mathrm{P} 2$, in conjunction with Proposition 3, suggest proposing the observer $\widehat{\Sigma}_{m_{T}}$ for the master system $\Sigma_{m_{T}}(11)$ as

$$
\begin{aligned}
\dot{\widehat{\mathbf{z}}}_{m}= & A_{n} \widehat{\mathbf{z}}_{m}+B_{n}\left(\phi_{m}\left(y_{m}\right)+K_{m}^{T} \widehat{\mathbf{z}}_{m}+f_{m}\left(\widehat{\mathbf{z}}_{m}\right)\right) \\
& -L_{n \boldsymbol{\kappa}_{m}}\left(y_{m}-C_{n}^{T} \widehat{\mathbf{z}}_{m}\right),
\end{aligned}
$$

where $y_{m}=z_{m_{1}}$. Similarly, observer $\widehat{\Sigma}_{s_{T}, u}$ for the slave system can be proposed as

$$
\begin{aligned}
\dot{\mathbf{z}}_{s}= & A_{n} \widehat{\mathbf{z}}_{s}+B_{n}\left(\phi_{s}\left(y_{s}\right)+K_{s}^{T} \widehat{\mathbf{z}}_{s}+f_{s}\left(\mathbf{z}_{s}\right)+p u_{s}\right) \\
& -L_{n \boldsymbol{\kappa}_{s}}\left(y_{s}-C_{n}^{T} \mathbf{z}_{s}\right)
\end{aligned}
$$

with $y_{s}=z_{s_{1}}$, where $L_{n \boldsymbol{\kappa}_{m}}$ and $L_{n \boldsymbol{\kappa}_{s}}$ must be selected, such that the following matrices are obtained:

$$
\begin{gathered}
M_{n \kappa_{m}}=A_{n}+B_{n} K_{m}^{T}-L_{n \kappa_{m}} C_{n}^{T} ; \\
M_{n \kappa_{s}}=A_{n}+B_{n} K_{s}^{T}-L_{n \kappa_{s}} C_{n}^{T},
\end{gathered}
$$

being Hurwitz and fulfilling the Lyapunov equation $P_{n \kappa_{i}} M_{n \kappa_{i}}+M_{n \kappa_{i}}^{T} P_{n \kappa_{i}}=-I_{n}, i=\{s, m\}$, with the following restrictions:

$$
\begin{aligned}
2 \gamma_{s}\left\|P_{n \kappa_{s}} B_{n}\right\| & <1 ; \\
2 \gamma_{m}\left\|P_{n \kappa_{m}} B_{n}\right\| & <1 .
\end{aligned}
$$

In the next section, we use the Lyapunov-based observer to solve the GS control problem. 
3.1. Generalized Synchronization. Now we are ready to introduce the main proposition of this paper.

Proposition 5. Assuming that the observers, (22) and (23), satisfy the conditions in Remark 4, the following static feedback,

$$
\begin{aligned}
& u\left(\widehat{\mathbf{z}}_{s}, \widehat{\mathbf{z}}_{m}\right)=\frac{1}{p}\left(-\bar{\phi}\left(y_{m}, y_{s}\right)-\bar{K}\left(\widehat{\mathbf{z}}_{m}, \widehat{\mathbf{z}}_{s}\right)-\bar{f}\left(\widehat{\mathbf{z}}_{m}, \widehat{\mathbf{z}}_{s}\right)\right. \\
& \left.\quad-K_{n, z}\left(\widehat{\mathbf{z}}_{m}-\widehat{\mathbf{z}}_{s}\right)\right),
\end{aligned}
$$

where,

$$
\begin{gathered}
\bar{\phi}\left(y_{m}, y_{s}\right) \hat{=} \phi_{m}\left(y_{m}\right)-\phi_{s}\left(y_{s}\right) ; \\
\bar{K}(\mathbf{x}, \mathbf{y}) \hat{=} K_{m}^{T} \mathbf{x}-K_{s}^{T} \mathbf{y} ; \\
\bar{f}(\mathbf{x}, \mathbf{y}) \hat{=} f_{m}(\mathbf{x})-f_{s}(\mathbf{y}),
\end{gathered}
$$

ensures that the synchronization error $e_{z}=z_{s}-z_{m}$ exponentially converges to zero.

Proof. From Assumptions P1 and P2, we have that systems $\Sigma_{m}$ and $\Sigma_{s, u}$ can be expressed according to (11) and (13), respectively. Computing the dynamic error, $\mathbf{e}_{z}$, we have

$$
\begin{aligned}
\dot{\mathbf{e}}_{z} & \\
= & A_{n} \mathbf{e}_{z} \\
& +B_{n}\left(\bar{\phi}\left(y_{m}, y_{s}\right)+\bar{K}\left(\mathbf{z}_{m}, \mathbf{z}_{s}\right)+\bar{f}\left(\mathbf{z}_{m}, \mathbf{z}_{s}\right)+p u\right) .
\end{aligned}
$$

Therefore, introducing $u\left(\widehat{\mathbf{z}}_{s}, \widehat{\mathbf{z}}_{m}\right)$, proposed in (26), into (28), we obtain

$$
\begin{aligned}
\dot{\mathbf{e}}_{z} & =A_{n} \mathbf{e}_{z}+B_{n}\left(-\bar{K}\left(\widehat{\mathbf{z}}_{m}, \widehat{\mathbf{z}}_{s}\right)+\bar{K}\left(\mathbf{z}_{m}, \mathbf{z}_{s}\right)+\Delta(\overline{\mathbf{z}})\right. \\
& \left.-K_{n, z}\left(\widehat{\mathbf{z}}_{m}-\widehat{\mathbf{z}}_{s}\right)\right),
\end{aligned}
$$

where $\overline{\mathbf{z}}=\left(\mathbf{z}_{m}^{T}, \mathbf{z}_{s}^{T}, \widehat{\mathbf{z}}_{m}^{T}, \widehat{\mathbf{z}}_{s}^{T}\right)$ and $\Delta$ are defined by

$$
\Delta(\overline{\mathbf{z}})=\bar{f}\left(\mathbf{z}_{m}, \mathbf{z}_{s}\right)-\bar{f}\left(\widehat{\mathbf{z}}_{m}, \widehat{\mathbf{z}}_{s}\right) .
$$

Remembering that $\mathbf{e}_{s}=\mathbf{z}_{s}-\widehat{\mathbf{z}}_{s}$ and $\mathbf{e}_{m}=\mathbf{z}_{m}-\widehat{\mathbf{z}}_{m}$, we have the following relations:

$$
\begin{aligned}
\bar{K} & \left(\widehat{\mathbf{z}}_{m}, \widehat{\mathbf{z}}_{s}\right)-\bar{K}\left(\mathbf{z}_{m}, \mathbf{z}_{s}\right) \\
& =K_{m}^{T} \widehat{\mathbf{z}}_{m}-K_{s}^{T} \widehat{\mathbf{z}}_{s}-K_{m}^{T} \mathbf{z}_{m}+K_{s}^{T} \mathbf{z}_{s} ; \\
& =-K_{m}^{T} \mathbf{e}_{m}+K_{s}^{T} \mathbf{e}_{s} .
\end{aligned}
$$

Similarly,

$$
K_{n, z}\left(\widehat{\mathbf{z}}_{m}-\widehat{\mathbf{z}}_{s}\right)=K_{n, z} \mathbf{e}_{z}+K_{n, z}\left(\mathbf{e}_{s}-\mathbf{e}_{m}\right) .
$$

Substituting these two relations into (29), we obtain

$$
\begin{aligned}
\dot{\mathbf{e}}_{z} & \\
= & \left(A_{n}-B_{n} K_{n, z}\right) \mathbf{e}_{z} \\
& +\overbrace{B_{n}\left(K_{n, z}\left(\mathbf{e}_{s}-\mathbf{e}_{m}\right)+K_{s}^{T} \mathbf{e}_{s}-K_{m}^{T} \mathbf{e}_{m}\right)+B_{n} \Delta(\overline{\mathbf{z}})}^{w(\mathbf{e}, \overline{\mathbf{z}})} .
\end{aligned}
$$

Using simple algebra, we have that $\Delta(\overline{\mathbf{z}})$ can be upper bounded, as follows:

$$
\begin{aligned}
\Delta & =\left|\bar{f}\left(\mathbf{z}_{m}, \mathbf{z}_{s}\right)-\bar{f}\left(\widehat{\mathbf{z}}_{m}, \widehat{\mathbf{z}}_{s}\right)\right| \\
& =\left|\left(f_{m}\left(\mathbf{z}_{m}\right)-f_{m}\left(\widehat{\mathbf{z}}_{m}\right)\right)-\left(f_{s}\left(\mathbf{z}_{s}\right)-f_{s}\left(\widehat{\mathbf{z}}_{s}\right)\right)\right| \\
& \leq \gamma_{m}\left|\mathbf{e}_{m}\right|+\gamma_{s}\left|\mathbf{e}_{s}\right| .
\end{aligned}
$$

Defining

$$
\bar{A}_{n s}=A_{n}-B_{n} K_{n, z}
$$

provided that $\bar{A}_{n s}$ is Hurwitz, (33) can be written in the following simple way:

$$
\dot{\mathbf{e}}_{z}=\bar{A}_{n s} \mathbf{e}_{z}+B_{n} w(\mathbf{e}, \overline{\mathbf{z}}),
$$

where $w(\mathbf{e}, \overline{\mathbf{z}})$ is given by

$$
\begin{aligned}
w(\mathbf{e}, \overline{\mathbf{z}})= & B_{n}\left(K_{n, z}\left(\mathbf{e}_{s}-\mathbf{e}_{m}\right)+K_{s}^{T} \mathbf{e}_{s}-K_{m}^{T} \mathbf{e}_{m}\right) \\
& +B_{n} \Delta(\overline{\mathbf{z}}) .
\end{aligned}
$$

However, according to (34), $w(*)$ can be upper bounded, as follows:

$$
\begin{aligned}
|w(*)| \leq & \left|K_{n, z}\right|\left\|\mathbf{e}_{s}-\mathbf{e}_{m}\right\|+\left|K_{s}^{T}\right|\left\|\mathbf{e}_{s}\right\|+\left|K_{m}^{T}\right|\left\|\mathbf{e}_{m}\right\| \\
& +\gamma_{m}\left|\mathbf{e}_{m}\right|+\gamma_{s}\left|\mathbf{e}_{s}\right| .
\end{aligned}
$$

By assumption, $\mathbf{e}_{s}$ and $\mathbf{e}_{m}$ converge exponentially and asymptotically to zero. Then, there are some $\alpha$ and $\beta$ being strictly positives, such that $|w(*)| \leq \alpha e^{-\beta t}$. Consequently, the error $\mathbf{e}_{z}$ of system (36) asymptotically and exponentially converges to zero.

\section{Numerical Simulations}

The goal of this example consists of solving the GS problem for the controlled slave Chua system and the free master Colpitts oscillator, provided that a single output of each system is available for measurements. On the other hand, we underscore that, in the present case, the trajectories of the Colpitts system are bounded in some compact set and exhibit chaotic or oscillatory behavior.

Master System. Consider the state equations for the Colpitts oscillator $\Sigma_{m}$, given by

$$
\begin{aligned}
& \dot{x}_{1}=-a_{m} e^{-x_{2}}+a_{m} x_{3}+a_{m}, \\
& \dot{x}_{2}=b_{m} x_{3}, \\
& \dot{x}_{3}=-c_{m} x_{1}-c_{m} x_{2}-d_{m} x_{3},
\end{aligned}
$$

where $a_{m}=b_{m}\left(C_{2} / C_{1}\right), b_{m}=I_{0} / w_{0} C_{2} V_{t}, c_{m}=V_{t} / w_{0} L I_{o}$, and $d_{m}=R / L w_{0}$ (to avoid confusion, the master parameters use the subscript $m$, and the slave parameters use the subscript $s)$. It is well known that this oscillator exhibits, for certain values of its parameters, a chaotic behavior. We will show that, 
selecting $y_{m}=x_{2}$ as the differential primitive element, we can write it according to expression (11). We use $\mathbf{x}_{m}^{T}=\left(x_{1}, x_{2}, x_{3}\right)$ to refer to the vector state of the Colpitts system. From $y_{m}=$ $x_{2}$, we obtain the following representation:

$$
\begin{aligned}
\mathbf{z}_{m} & =\Phi_{m}\left(\mathbf{x}_{m}\right)=\left[\begin{array}{c}
y_{m} \\
\dot{y}_{m} \\
\ddot{y}_{m}
\end{array}\right] \\
& =\left[\begin{array}{c}
x_{2} \\
b_{m} x_{3} \\
b_{m}\left(-c_{m} x_{1}-c_{m} x_{2}-d_{m} x_{3}\right)
\end{array}\right],
\end{aligned}
$$

where $\Phi_{m}$ is invertible. Furthermore, the time derivative of $\mathbf{z}_{m}$ can be written as

$$
\left[\begin{array}{c}
\dot{z}_{m_{1}} \\
\dot{z}_{m_{2}} \\
\dot{z}_{m_{3}}
\end{array}\right]=\left[\begin{array}{c}
z_{m_{2}} \\
z_{m_{3}} \\
\Psi_{n}\left(\mathbf{x}_{m}\right)
\end{array}\right]
$$

where

$$
\Psi_{m}\left(\mathbf{x}_{m}\right)=-b_{m} c_{m} \dot{x}_{1}-b_{m} c_{m} \dot{x}_{2}-b_{m} d_{m} \dot{x}_{3}
$$

Notice that the term $\Psi_{m}\left(\mathbf{x}_{m}\right)$ can be expressed in the coordinates of $\mathbf{z}_{m}$, as

$$
\begin{aligned}
\Psi_{m}\left(\mathbf{z}_{m}\right)= & \overbrace{a_{m} b_{m} c_{m}\left(-1+e^{-y_{m}}\right)}^{\phi_{m}\left(y_{m}\right)} \\
& +\overbrace{\left(-a_{m} c_{m}-b_{m} c_{m}\right) z_{2_{m}}-d_{m} z_{3_{m}}}^{K_{m}^{T} \mathbf{z}_{m}} .
\end{aligned}
$$

Notice that (41) and (43) are in agreement with the condition in Assumption P1. According to Proposition 3 and Remark 4, the observer can be proposed as

$$
\begin{aligned}
\dot{\mathbf{z}}_{m}= & A_{3} \widehat{\mathbf{z}}_{m}+B_{3}\left(\phi_{m}\left(y_{m}\right)+K_{m}^{T} \widehat{\mathbf{z}}_{m}\right) \\
& -L_{3 \boldsymbol{\kappa}_{m}} C_{3}^{T}\left(\widehat{\mathbf{z}}_{m}-\mathbf{z}_{m}\right)
\end{aligned}
$$

where $L_{3 \boldsymbol{\kappa}_{m}}$ has to be selected, such that $M_{3 \boldsymbol{\kappa}_{m}}=\left(A_{3}+B_{3} K_{m}^{T}-\right.$ $\left.L_{3 \kappa_{m}} C_{3}^{T}\right)$ is Hurwitz. Using the following actual parameters reported in [27],

$$
\begin{aligned}
& a=b=6.2723, \\
& c=0.0797, \\
& d=0.6898,
\end{aligned}
$$

it is easy to show that selecting $L_{3 \kappa_{\mathrm{s}}}=[6,11,6]$ allows us to obtain the following matrix:

$$
M_{3 \kappa_{m}}=\left[\begin{array}{ccc}
-6 & 1 & 0 \\
-11 & 0 & 1 \\
-6 & -0.249 & -0.689
\end{array}\right]
$$

which is Hurwitz. Therefore, proposed observer (44) ensures that the estimation error $\mathbf{e}_{m}=\mathbf{z}_{m}-\widehat{\mathbf{z}}_{m}$ converges exponentially and asymptotically to zero.

For the numerical simulation, the experimental setup assumed the above defined parameter values, the master initial conditions as $x_{m}(0)=(1,-1,2)$, and the master observer as $\hat{x}_{m}(0)=0$. The obtained results are shown in Figure 1, where we can see that the estimation error of each state variable settles to almost zero after nearly 4 seconds.

Slave System. Consider the Chua chaotic system as the controlled slave system, $\Sigma_{s, u}$. This system is formed by three linear energy-storage elements (an inductor and two capacitors), a linear resistor, and a single nonlinear resistor. It is well known that this circuit is described by the following differential equations:

$$
\begin{aligned}
& \dot{x}_{1}=a_{s}\left(x_{2}-x_{1}-v_{s}\left(x_{1}\right)\right)+u, \\
& \dot{x}_{2}=x_{1}-x_{2}+x_{3}, \\
& \dot{x}_{3}=-b_{s} x_{2},
\end{aligned}
$$

with

$$
v_{s}(x)=m_{1} x+\frac{m_{0}-m_{1}}{2}(|x+1|-|x-1|),
$$

where parameters $a_{s}, b_{s}, m_{0}$, and $m_{1}$ are chosen, such that system (47) exhibits a chaotic behavior when $u=0$. Notice that $u$ is the input of the slave system that is necessary to achieve the GS problem. Particularly, in the case where $u=0$ and the parameters values are in the neighbourhood of [28],

$$
\begin{aligned}
b_{s} & =28 \\
a_{s} & =15.6 ; \\
m_{0} & =-\frac{8}{7} \\
m_{1} & =-\frac{5}{7}
\end{aligned}
$$

it is known that we have the so-called double scroll chaotic attractors. As before, we use symbol $\mathbf{x}_{s}^{T}=\left(x_{1}, x_{2}, x_{3}\right)$ to indicate the states of slave system (47). In a similar fashion, we choose as the primitive element $y_{s}=x_{3}$. Therefore, to obtain the corresponding canonical form, we derive $y_{s}$ two times, obtaining

$$
\mathbf{z}_{s}=\Phi_{s}\left(\mathbf{x}_{s}\right)=\left[\begin{array}{c}
y_{s} \\
\dot{y}_{s} \\
\ddot{y}_{s}
\end{array}\right]=\left[\begin{array}{c}
x_{3} \\
-b x_{2} \\
-b_{s}\left(x_{1}-x_{2}+x_{3}\right)
\end{array}\right],
$$

where $\Phi_{s}$ is an invertible map. From the above equation, the time derivative of $\mathbf{z}_{s}$ is given by

$$
\left[\begin{array}{c}
\dot{z}_{s_{1}} \\
\dot{z}_{s_{2}} \\
\dot{z}_{s_{3}}
\end{array}\right]=\left[\begin{array}{c}
z_{s_{2}} \\
z_{s_{3}} \\
\Psi_{s}\left(\mathbf{x}_{s}\right)-b_{s} u
\end{array}\right]
$$



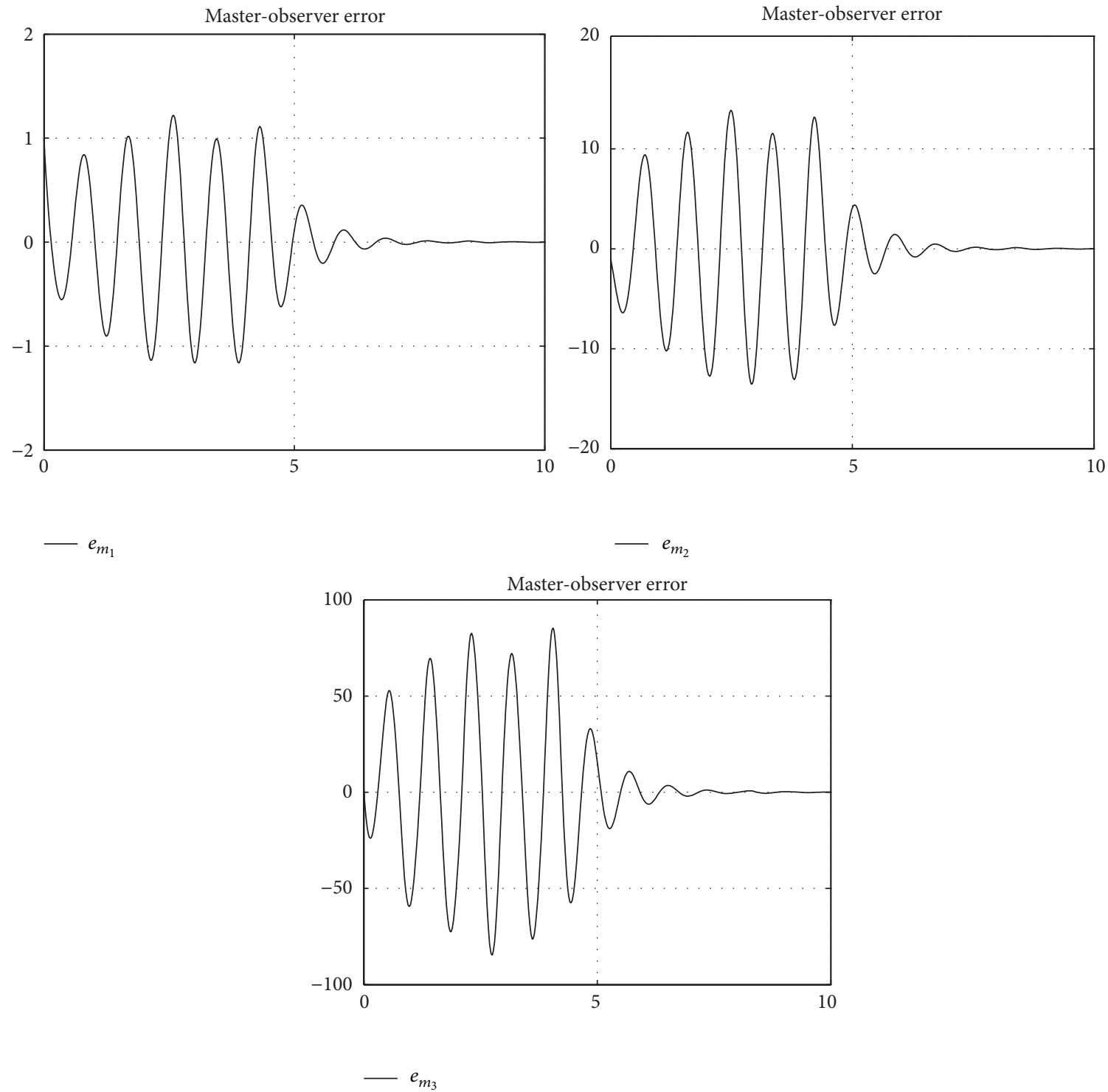

FIGURE 1: Observation error for the Colpitts or master system in the transformed coordinates: $e_{m}=z_{m}-\widehat{z}_{m}$.

where

$$
\Psi_{s}\left(\mathbf{x}_{s}\right)=-b_{s}\left(\dot{x}_{1}-\dot{x}_{2}+\dot{x}_{3}\right) .
$$

Notice that $\Psi_{s}\left(\mathbf{x}_{s}\right)$ can be rewritten in the coordinates of $\mathbf{z}_{s}$ as

$$
\Psi_{s}\left(y_{s}, \mathbf{z}_{s}\right)=K_{s}^{T} \mathbf{z}_{s}+a_{s} b_{s} f_{s}\left(x_{1}\right)-b_{s} u,
$$

where $f_{s}\left(x_{1}\right)=v_{x}\left(x_{1}\right)$ and

$$
\begin{gathered}
x_{1}=-z_{s_{1}}-\frac{z_{s_{2}}+z_{s_{3}}}{b} ; \\
K_{s}^{T}=\left[\begin{array}{lll}
-a b & -b-1-a
\end{array}\right] .
\end{gathered}
$$

On the other hand, it is clear that $v_{x}$ is Lipchitz, with $\gamma_{s}=$ $\left|m_{1}\right|+\left|m_{0}-m_{1}\right|$ (see Appendix). Notice that (51) and (53) are in agreement with Assumption P2. Now, according to Proposition 3 and Remark 4, the observer can be proposed as

$$
\begin{aligned}
\dot{\mathbf{z}}_{s}= & A_{3} \widehat{\mathbf{z}}_{s}+B_{3}\left(a b v_{x}\left(x_{1}\right)-b u+K_{s}^{T} \widehat{\mathbf{z}}_{s}\right) \\
& -L_{3 \boldsymbol{\kappa}_{s}} C_{3}^{T}\left(\widehat{\mathbf{z}}_{s}-\mathbf{z}_{s}\right) .
\end{aligned}
$$

Using $L_{3 \boldsymbol{\kappa}_{s}}=[24,26,9]$ and the list of data (49), we have the following matrix:

$$
M_{3 \boldsymbol{\kappa}_{s}}=\left[\begin{array}{ccc}
-24 & 1 & 0 \\
-26 & 0 & 1 \\
-399 & -25 & -16
\end{array}\right] \text {, }
$$



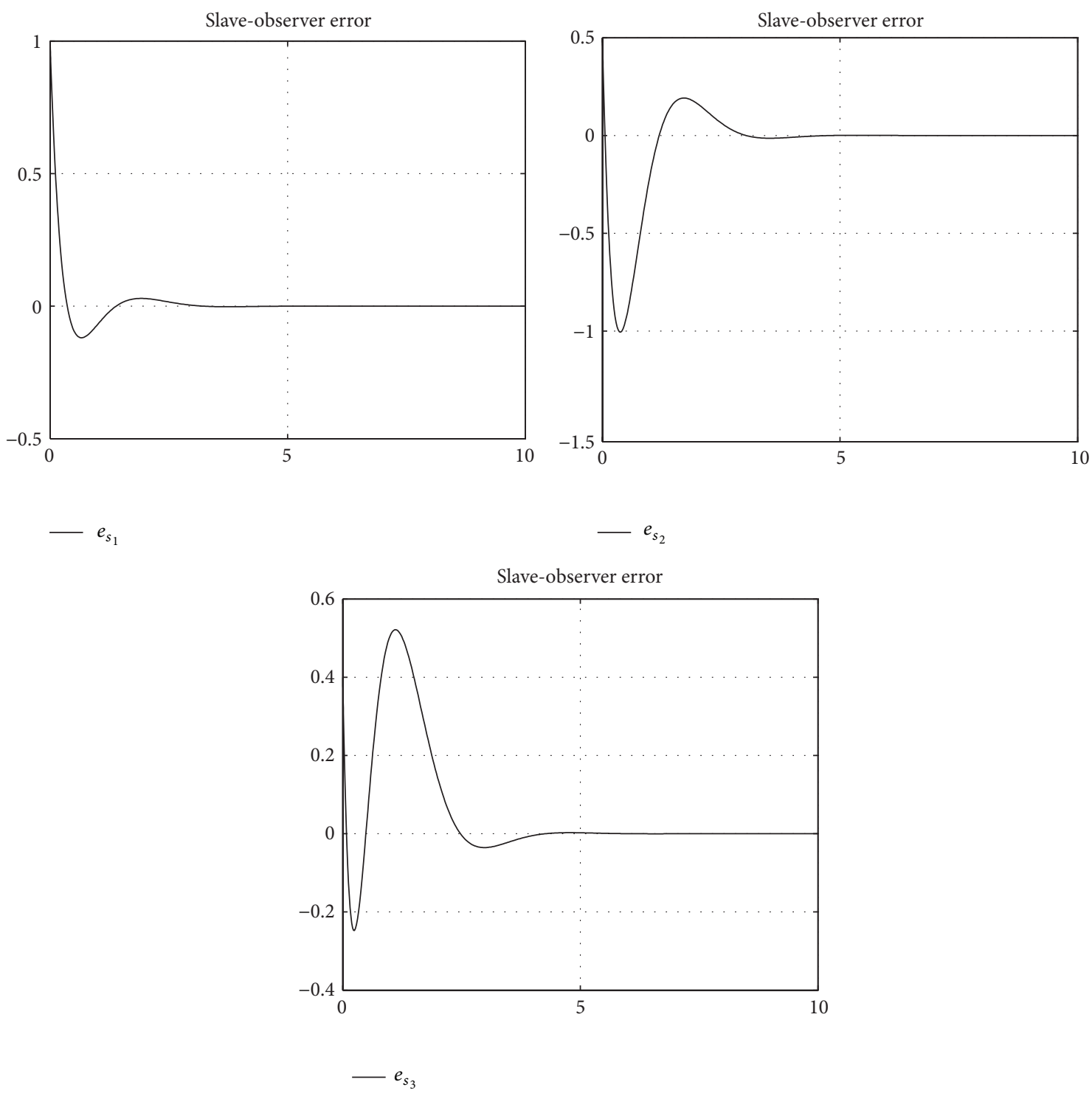

Figure 2: Observation error for the Chua or slave system in the transformed coordinates: $e_{s}=z_{s}-\widehat{z}_{s}$.

which is stable, and $\gamma_{s}=1.1429$. Solving the Lyapunov equation,

$$
P_{3 \kappa_{s}} M_{3 \kappa_{s}}+M_{3 \kappa_{s}}^{T} P_{3 \kappa_{s}}=-I_{3},
$$

it is easy to see that $\left\|P_{3 \kappa_{\mathrm{s}}} B_{3}\right\|=0.308$, ensuring that $2 \gamma_{s}(0.308)<1$. That is, the corresponding observer proposed in (55) ensures that the estimation error $\mathbf{e}_{s}=\mathbf{z}_{s}-\widehat{\mathbf{z}}_{s}$ converges exponentially and asymptotically to zero.

Similarly to the master system, the slave experimental setup uses the above defined parameter values, and the initial conditions of the slave and its observer were fixed as $x_{s}(0)=$ $(1,0.5,0.4)$ and $\hat{x}_{s}(0)=0$, respectively. The slave input was fixed as $u=0$. In Figure 2 , it is easy to see that the errors are very close to zero after 3.5-second elapse.

Colpitts Oscillator and Chua Circuit Generalized Synchronization. For this numerical simulation, we used the same experimental setup and the same observer as in the previous experiments. The setup for the slave controller was fixed according to Proposition 5. The used static feedback was

$$
\begin{aligned}
& u\left(\widehat{\mathbf{z}}_{s}, \widehat{\mathbf{z}}_{m}\right)=\frac{1}{p}\left(-\bar{\phi}\left(y_{m}\right)-\bar{K}\left(\widehat{\mathbf{z}}_{m}, \widehat{\mathbf{z}}_{s}\right)-\bar{f}\left(\mathbf{z}_{s}\right)\right. \\
& \left.\quad-K_{n, z}\left(\widehat{\mathbf{z}}_{m}-\widehat{\mathbf{z}}_{s}\right)\right),
\end{aligned}
$$

where

$$
\begin{aligned}
\bar{\phi}\left(y_{m}\right) & =a_{m} b_{m} c_{m}\left(-1+e^{-y_{m}}\right) ; \\
\bar{K}\left(\widehat{\mathbf{z}}_{m}, \widehat{\mathbf{z}}_{s}\right) & =K_{m}^{T} \widehat{\mathbf{z}}_{m}-K_{s}^{T} \widehat{\mathbf{z}}_{s} ; \\
\bar{f}\left(\mathbf{z}_{s}\right) & =-a_{s} b_{s} v_{s}\left(-z_{s_{1}}-\frac{z_{s_{2}}+z_{s_{3}}}{b_{s}}\right) .
\end{aligned}
$$

The synchronization error of this experiment is shown in Figure 3, where we can see that, after 9-second elapse, the difference between each pair of transformed coordinates is almost zero, for the case $r=1$. 

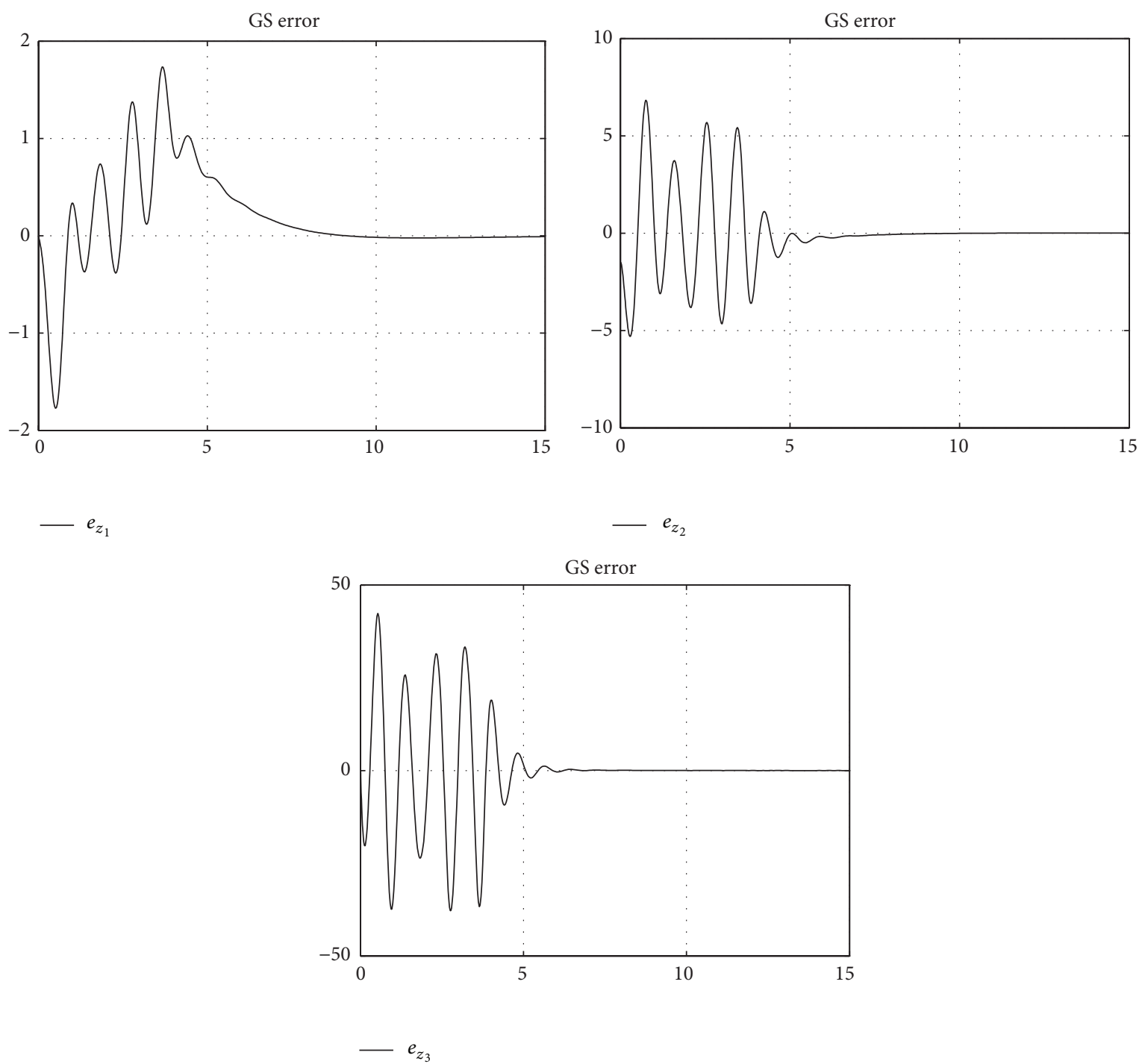

FIGURE 3: Generalized synchronization error between the free Colpitts oscillator and the driven Chua circuit in transformed coordinates.

\section{Conclusions}

In this paper, we proposed a solution to the output-feedback GS problem for a class of chaotic systems, namely, the free master and the controlled slave, assuming that the master system trajectories are bounded in a compact set. The problem was solved given that the slave was controlled using a single input, and a single output of each system was available or measurable. The solution consisted of expressing both systems in their corresponding observable generalized canonical form, through the differentiable primitive element. To this end, a suitable diffeomorphism transformation was applied, between the original state space and the corresponding canonical observability form of each system. Due to the fact that the controlled slave system uses its own whole state and the whole state of the master, a kind of a Luenberger observer for each system was proposed. Finally, the information obtained through the observers is used by the controlled slave system, solving the GS problem. The corresponding convergence analysis was carried out using the linear control approach and the Lyapunov method. The effectiveness of the obtained method was assessed by convincing numerical simulations, using the chaotic Colpitts oscillator as the master and the chaotic Chua circuit as the slave.

\section{Appendix}

\section{Computing the Lipchitz Constant for Function $v_{x}$}

From the definition given in (48), we have that

$$
\begin{aligned}
& \left|v_{x}(x)-v_{x}(y)\right| \\
& \leq\left|m_{1}\right||x-y|+\frac{\left|m_{0}-m_{1}\right|}{2}|(|x+1|-|y+1|)| \\
& \quad+\frac{\left|m_{0}-m_{1}\right|}{2}|(|y-1|-|x-1|)| .
\end{aligned}
$$


After using the well-known inequality,

$$
|(|a|-|b|)| \leq|a-b|,
$$

(A.1) can be rewritten as

$$
\begin{aligned}
\left|v_{x}(x)-v_{x}(y)\right| & \leq\left|m_{1}\right||x-y|+\left|m_{0}-m_{1}\right||x-y| \\
& \leq \bar{m}|x-y|
\end{aligned}
$$

where

$$
\bar{m}=\left|m_{1}\right|+\left|m_{0}-m_{1}\right| \text {. }
$$

\section{Conflict of Interests}

The authors declare that there is no conflict of interests regarding the publication of this paper.

\section{Acknowledgments}

This research was supported by the Secretaría de Investigación y Posgrado of the Instituto Politécnico Nacional (SIP-IPN) under Research Grants 20150929 and 20150351 and by the Departamento de Control Automático of the CINVESTAV-IPN.

\section{References}

[1] T. Yang and L. O. Chua, "Channel-independent chaotic secure communication," International Journal of Bifurcation and Chaos in Applied Sciences and Engineering, vol. 6, no. 12, pp. 26532660, 1996.

[2] O. I. Moskalenko, A. A. Koronovskii, and A. E. Hramov, "Generalized synchronization of chaos for secure communication: remarkable stability to noise," Physics Letters A, vol. 374, no. 29, pp. 2925-2931, 2010.

[3] X. Jiang-Feng, M. Le-Quan, and C. Guan-Rong, "A chaotic communication scheme based on generalized synchronization and hash functions," Chinese Physics Letters, vol. 21, no. 8, article 1445, 2004.

[4] L.-Q. Min, G.-R. Chen, X.-D. Zhang, X.-H. Zhang, and M. Yang, "Approach to generalized synchronization with application to chaos-based secure communication," Communications in Theoretical Physics, vol. 41, no. 4, pp. 632-640, 2004.

[5] H. D. I. Abarbanel, N. F. Rulkov, and M. M. Sushchik, "Generalized synchronization of chaos: the auxiliary system approach," Physical Review E, vol. 53, no. 5, pp. 4528-4535, 1996.

[6] N. F. Rulkov, M. M. Sushchik, L. S. Tsimring, and H. D. I. Abarbanel, "Generalized synchronization of chaos in directionally coupled chaotic systems," Physical Review E, vol. 51, no. 2, pp. 980-994, 1995.

[7] S. S. Yang and C. K. Duan, "Generalized synchronization in chaotic systems," Chaos, Solitons \& Fractals, vol. 9, no. 10, pp. 1703-1707, 1998.

[8] B. R. Hunt, E. Ott, and J. A. Yorke, "Differentiable generalized synchronization of chaos," Physical Review E: Statistical, Nonlinear, and Soft Matter Physics, vol. 55, no. 4, pp. 4029-4034, 1997.

[9] A. E. Hramov and A. A. Koronovskii, "Generalized synchronization: a modified system approach," Physical Review E, vol. 71, no. 6, Article ID 067201, 2005.
[10] J. R. Terry and G. D. VanWiggeren, "Chaotic communication using generalized synchronization," Chaos, Solitons \& Fractals, vol. 12, no. 1, pp. 145-152, 2001.

[11] A. E. Hramov and A. A. Koronovskii, "Intermittent generalized synchronization in unidirectionally coupled chaotic oscillators," Europhysics Letters, vol. 70, no. 2, pp. 169-175, 2005.

[12] G. Zhang, Z. Liu, and Z. Ma, "Generalized synchronization of different dimensional chaotic dynamical systems," Chaos, Solitons \& Fractals, vol. 32, no. 2, pp. 773-779, 2007.

[13] J. Lu and Y. Xi, "Linear generalized synchronization of continuous-time chaotic systems," Chaos, Solitons \& Fractals, vol. 17, no. 5, pp. 825-831, 2003.

[14] A. E. Matouk, "Chaos synchronization between two different fractional systems of Lorenz family," Mathematical Problems in Engineering, vol. 2009, Article ID 572724, 11 pages, 2009.

[15] Q. Bian and H. Yao, "Generalized synchronization between two complex dynamical networks with time-varying delay and nonlinear coupling," Mathematical Problems in Engineering, vol. 2011, Article ID 978612, 15 pages, 2011.

[16] Y. Li, Y. Zhao, and Z.-A. Yao, "Chaotic control and generalized synchronization for a hyperchaotic lorenz-stenflo system," Abstract and Applied Analysis, vol. 2013, Article ID 515106, 18 pages, 2013.

[17] T. Yang and L. O. Chua, "Generalized synchronization of chaos via linear transformations," International Journal of Bifurcation and Chaos in Applied Sciences and Engineering, vol. 9, no. 1, pp. 215-219, 1999.

[18] A. Kittel, J. Parisi, and K. Pyragas, "Generalized synchronization of chaos in electronic circuit experiments," Physica D: Nonlinear Phenomena, vol. 112, no. 3-4, pp. 459-471, 1998.

[19] W. Liu, X. Qian, J. Yang, and J. Xiao, "Antisynchronization in coupled chaotic oscillators," Physics Letters, Section A: General, Atomic and Solid State Physics, vol. 354, no. 1-2, pp. 119-125, 2006.

[20] E. R. Kolchin, Differential Algebra \& Algebraic Groups, Volume 54, Academic Press, 1973.

[21] R. Martínez-Guerra and J. L. Mata-Machuca, "Generalized synchronization via the differential primitive element," Applied Mathematics and Computation, vol. 232, pp. 848-857, 2014.

[22] M. Fliess and H. Sira-Ramirez, "Control via state estimations of some nonlinear systems," in Proceedings of the 6th IFAC Symposium on Nonlinear Control Systtems (NOLCOS '04), Stuttgart, Germany, September 2004.

[23] M. Fliess, "Generalized controller canonical forms for linear and nonlinear dynamics," IEEE Transactions on Automatic Control, vol. 35, no. 9, pp. 994-1001, 1990.

[24] R. Martínez-Guerra, C. A. Pérez-Pinacho, and G. C. GómezCortés, "Synchronization of chaotic liouvillian systems: an application to Chua's oscillator," in Synchronization of Integral and Fractional Order Chaotic Systems, Understanding Complex Systems, pp. 135-151, Springer, 2015.

[25] H. Sira-Ramirez and S. K. Agrawal, Differentially Flat Systems, Volume 17, CRC Press, 2004.

[26] Y.-W. Wang and Z.-H. Guan, "Generalized synchronization of continuous chaotic system," Chaos, Solitons \& Fractals, vol. 27, no. 1, pp. 97-101, 2006. 
[27] J. L. Mata-Machuca and R. Martínez-Guerra, "Asymptotic synchronization of the Colpitts oscillator," Computers \& Mathematics with Applications, vol. 63, no. 6, pp. 1072-1078, 2012.

[28] H. Sira-Ramírez, A. Luviano-Juárez, and J. Cortés-Romero, "Flatness-based linear output feedback control for disturbance rejection and tracking tasks on a Chua's circuit," International Journal of Control, vol. 85, no. 5, pp. 594-602, 2012. 


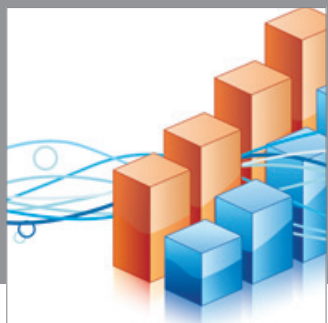

Advances in

Operations Research

mansans

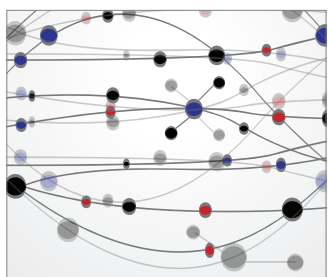

The Scientific World Journal
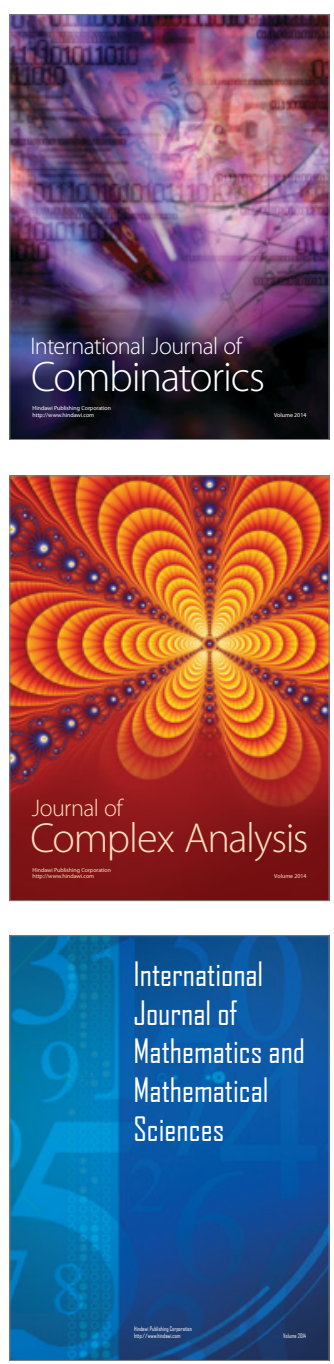
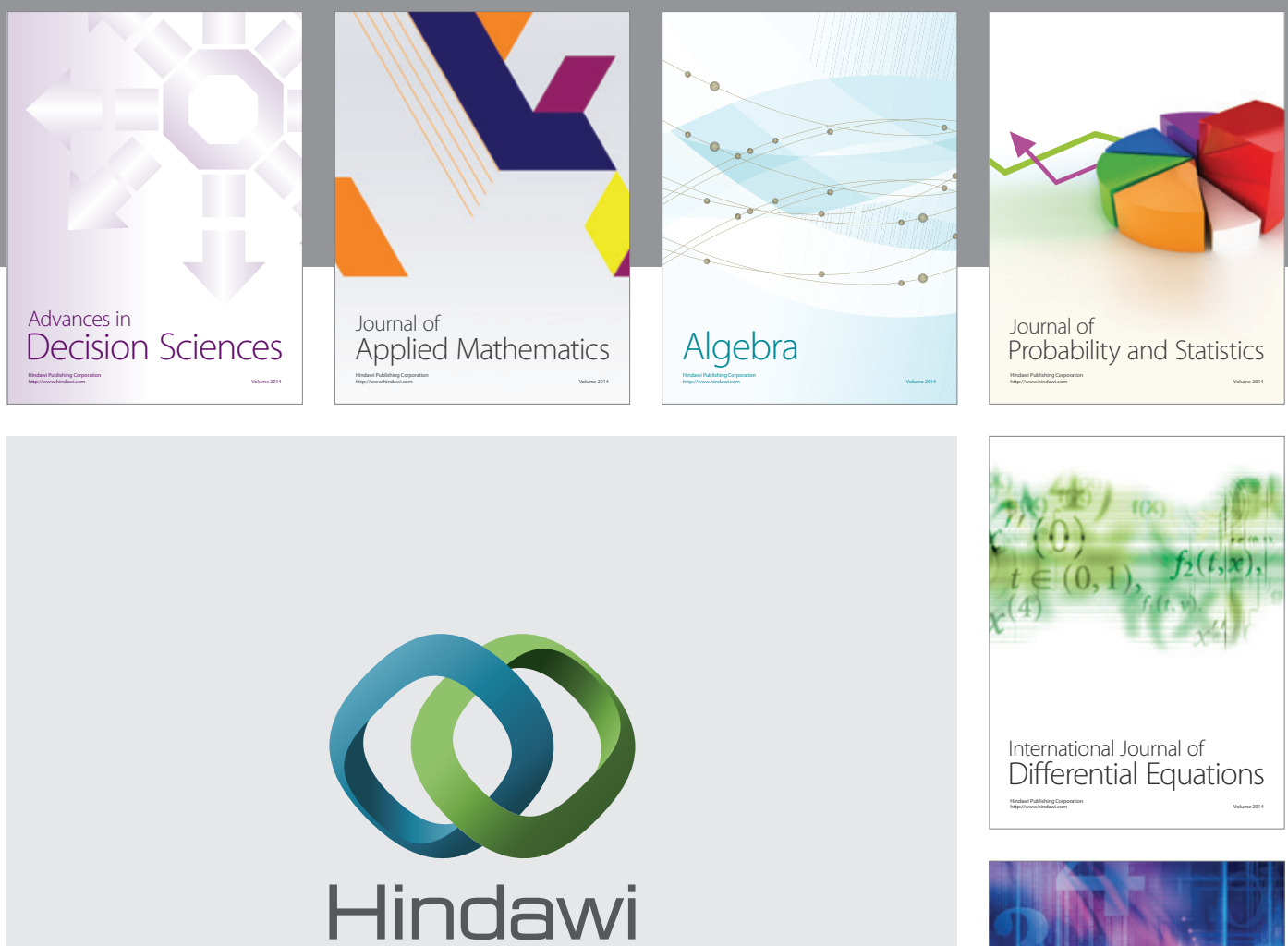

Submit your manuscripts at http://www.hindawi.com
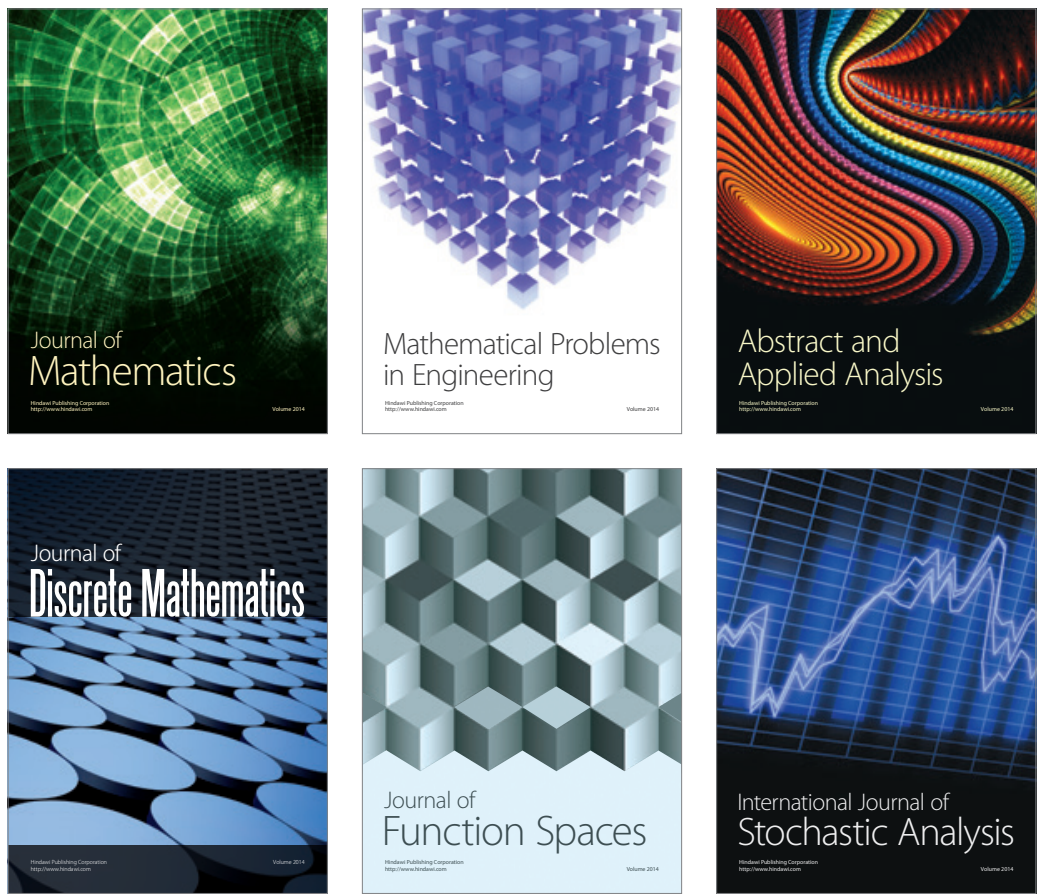

Journal of

Function Spaces

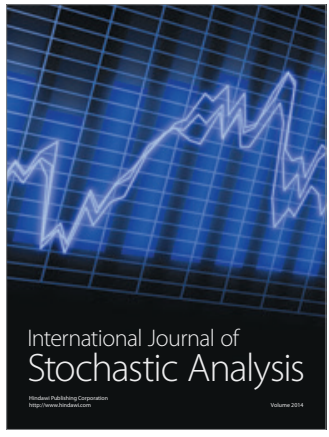

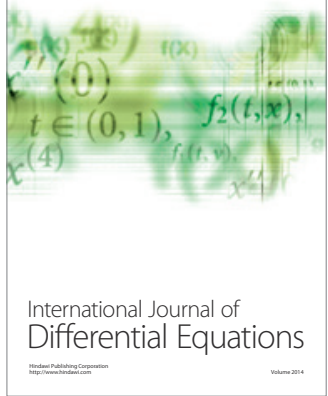
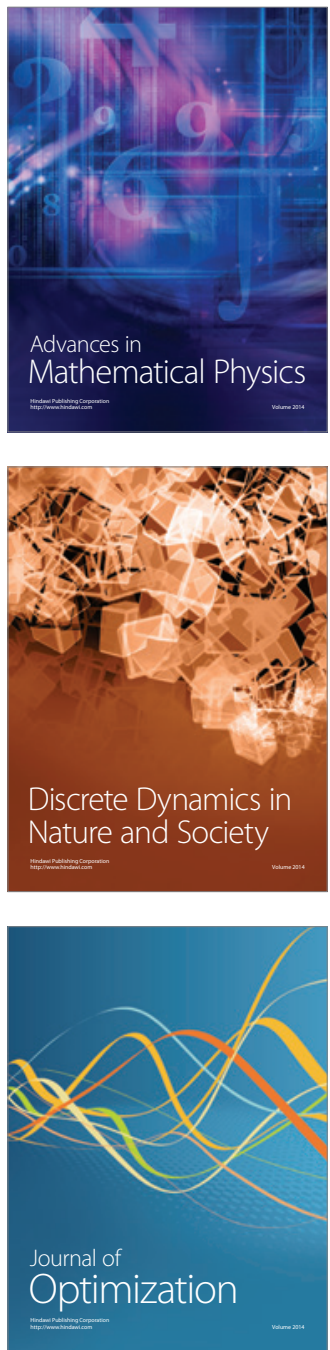